\title{
Psychological and Behavioral Abnormalities as the Initial Manifestation of Idiopathic Hypoparathyroidism: A Case Report
}

Pingping Ning, Xinglong Yang, Quanzhen Zhao, Hongyan Huang, Ran An, Yalan Chen, Yanming $\mathrm{Xu}^{\dagger}$

\begin{abstract}
Background

Idiopathic hypoparathyroidism is a rare endocrine disorder typically manifesting as paresthesias of the extremities and perioral region, muscle cramps, capopedal spasms, tetany, seizure, and fatigue. The condition is not known to manifest initially as psychological and behavioral abnormalities.
\end{abstract}

\section{Case presentation}

We report the case of a 53-year-old Chinese woman who presented with symptoms of depression, anxiety, feelings of agitation, irritability, hyperhidrosis, slow action and decreased interest in most activities. Computed tomography of the brain revealed intracranial calcification, scores on the Hamilton Anxiety Scale and Hamilton Depression Scale showed that she suffered from depression and anxiety, and scores on the Minimum Mental State Examination and Montreal Cognitive Assessment revealed impaired cognitive function. Laboratory analyses showed hypocalcemia, hyperphosphatemia and parathyroid hormone deficiency. Calcium carbonate and vitamin D3 supplementation therapy improved the patient's clinical symptoms of depression and anxiety, as well as her levels of calcium and phosphate. However, this treatment did not substantially improve the level of parathyroid hormone or reduce cognitive impairment.

\section{Conclusions}

Idiopathic hypoparathyroidism can manifest initially as psychological and behavioral abnormalities, which clinicians should keep in mind.

\section{Keywords:}

Psychological and behavioral abnormalities, Idiopathic hypoparathyroidism, Intracranial calcification, Hypocalcemia, Hyperphosphatemia, Parathyroid hormone deficiency

\begin{tabular}{ll}
\hline Abbreviations: & $\begin{array}{l}\text { disease characterized by hypocalcemia, } \\
\text { hyperphosphatemia and inappropriately low }\end{array}$ \\
MMSE: Minimum Mental State Examination; & $\begin{array}{l}\text { serum parathyroid hormone [1]. Its incidence } \\
\text { in the US is approximately 24-37 per 100,000 } \\
\text { porson-years, and approximately } 75 \% \text { of affected } \\
\text { individuals are female [2]. The condition most } \\
\text { Background }\end{array}$ \\
often results from anterior neck surgery, such as
\end{tabular}


thyroidectomy, parathyroidectomy, and head or neck cancer surgery. Less often, the disease has non-surgical origins and is referred to as hypomagnesemia, pseuodohypoparathyroidism, and idiopathic hypoparathyroidism.

The presenting symptoms of hypoparathyroidism are variable and usually result from the low serum level of calcium. Typical manifestations include paresthesias of the extremities and perioral region, muscle cramps, carpopedal spasms, tetany, seizure, and fatigue [3]. A few patients with hypoparathyroidism, especially idiopathic disease, have psychological and behavioral abnormalities, which can pose a great challenge to diagnosis. The literature contains reports of secondary hypoparathyroidism accompanying mental disorders, but we are unaware of reports of idiopathic hypoparathyroidism initially manifesting as psychological and behavioral abnormalities [4-8].

Here we describe a case of a patient with idiopathic hypoparathyroidism presenting only psychological and behavioral abnormalities was not found in the scientific literature until the time of writing this work. This case report should alert clinicians and psychiatrists to the possibility of hypoparathyroidism in the absence of secondary causes of parathyroid hormone deficiency, even when no typical manifestations precede the onset of psychiatric symptoms.

\section{Case Presentation}

A 53-year-old Chinese woman complaining of psychological and behavioral abnormalities lasting for five years was admitted to our hospital. She reported experiencing depression, anxiety, feelings of agitation, irritability, hyperhidrosis, and decreased interest in most activities. Her family reported personality disturbances and mood swings; for example, she could not control her emotions and often cried over trivial things. Prior to these complaints, the patient did not suffer from muscle cramps, carpopedal spasms, tetany, hypocalcemia-related seizures or paresthesias involving the perioral region and finger tips. Through our investigation, we found that the patient had never had mental illness before, and it was not present among her relatives. Psychiatric examination indicated abulia, emotional irritability, involuntary crying, unsociability and lethargy. No evidence of hallucinations or delusions was found. Examination of advanced neurological functions revealed reduced calculation, comprehension and executive abilities. Physical examination showed active tendon reflexes involving the four limbs, and bilateral Babinski and Chaddock signs were positive. No sensory or motor deficits were found, and Chvostek and Trousseau signs were absent. Signs suggesting pseudohypoparathroidism were absent, including cataracts, facial dysmorphism, goiter, metacarpal/metatarsal shortening, or other skeletal abnormalities. She was a junior high school graduate. The Minimum Mental State Examination (MMSE) showed mild damage (26 scores; total, 30 scores), the Montreal Cognitive Assessment (MoCA) suggested moderate cognitive impairment (18 scores; total, 30 scores), and the Hamilton Anxiety Scale suggested anxiety (15 scores). The Hamilton Depression Scale (17 items) indicated moderate depression (18 scores). Brain computed tomography showed bilateral symmetrical calcifications involving the caudate nucleus, globus pallidus, putamen, cerebellum, and subcortex (Figure 1). Laboratory tests showed low total calcium level (2.04 mmol/l; normal, 2.1-2.7 mmol/l), elevated serum inorganic phosphate level $(1.59 \mathrm{mmol} / \mathrm{l}$; normal,

0.81-1.45 $\mathrm{mmol} / \mathrm{l})$, low 25-hydroxyvitamin D (18.77 nmol/l; normal, 47.7-144 nmol/l) and low parathyroid hormone $(1.06 \mathrm{pmol} / \mathrm{l}$; normal, 1.60-1.90 pmol/l). Serum levels of magnesium, thyroid-stimulating hormone, T3 and T4 thyroid hormones, creatinine, blood urea nitrogen, and albumin were normal. Renal functions were also normal. The patient had never taken omeprazole.

The patient was diagnosed with idiopathic hypoparathyroidism initially presenting as psychological and behavioral abnormalities. This diagnosis was based on the findings of hypocalcemia, hyperphosphatemia, parathyroid hormone deficiency, normal serum magnesium, normal renal function, and computed tomography showing multiple intracranial calcifications. The patient was given calcium carbonate (1.5 g/day) and vitamin D3 (120 IU/ day). During the 15-day hospitalization, her symptoms of depression and anxiety improved, while her mood stabilized to some extent after total serum calcium levels returned to the normal range. For example, her words and facial expressions getting better. Also, when we talked with her, she didn't seem to be intense as she was on admission. However, cognitive function did not significantly improve. 
Psychological and Behavioral Abnormalities as the Initial Manifestation of Idiopathic Case Report Hypoparathyroidism: A Case Report
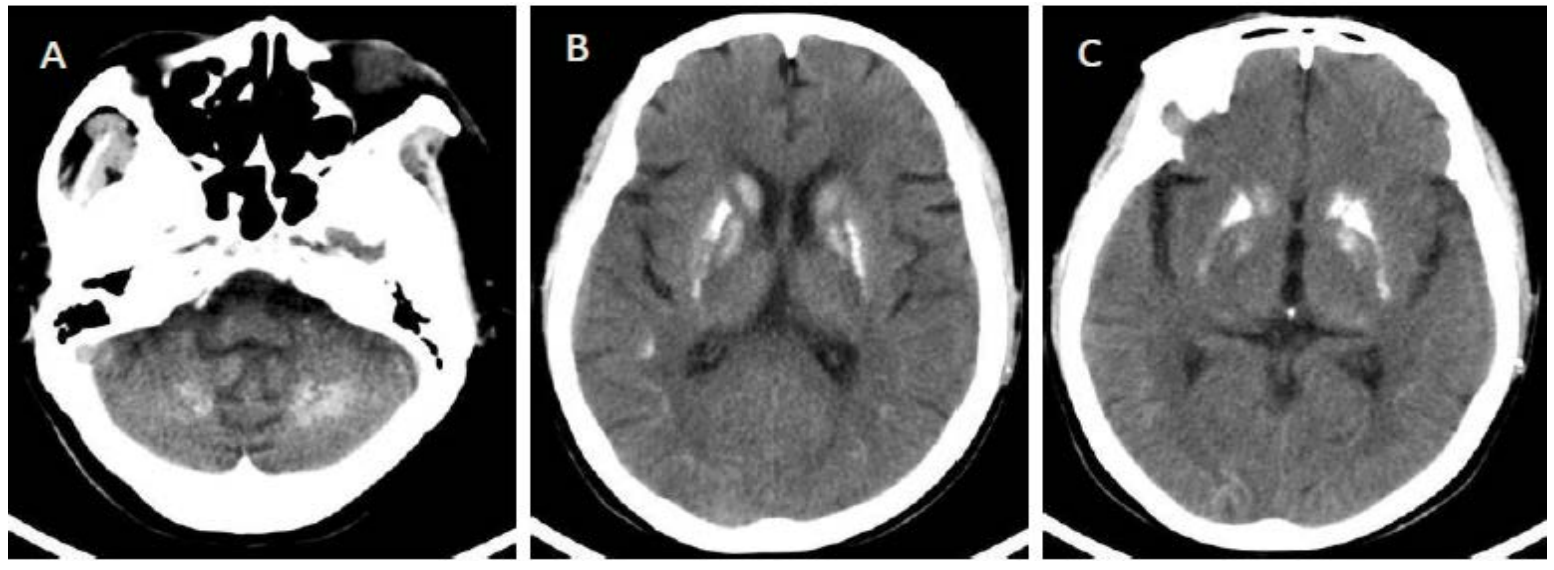

Figure 1: Calcification detected by brain computed tomography. Tomograms revealed bilateral symmetrical calcifications (A) in the cerebellum and (B-C) throughout the basal ganglia and subcortical regions.

The patient was discharged with a normal level of total serum calcium $(2.31 \mathrm{mmol} / \mathrm{l})$ and a slightly elevated serum inorganic phosphate level (1.53 $\mathrm{mmol} / \mathrm{l})$. Her clinical improvement persisted based on findings at a routine outpatient visit at 2 months after hospital discharge. At that visit, levels of total calcium and serum inorganic phosphate were within the normal range. However, the parathyroid hormone level was low, the MMSE indicated mild damage and MoCA showed moderate cognitive impairment.

\section{Discussion}

Our patient was diagnosed with hypoparathyroidism based on low calcium, high phosphate and low parathyroid hormone [9]. Her hypoparathroidism seems unlikely to be the result of secondary factors because her renal function and magnesium level were normal, she had never taken medications linked to parathyroid hormone deficiency, and she did not report cataracts, facial dysmorphism, goiter, metacarpal/metatarsal shortening, or other skeletal abnormalities suggestive of pseudohypoparathroidism. We also considered Fahr's disease when we first saw the patient's head Computed Tomography image. However, we all know that Fahr's disease which refers to the idiopathic basal ganglia calcification. There will be no secondary cause found in Fahr's disease and the laboratory examinations of these patients showed that serum levels of calcium, phosphorus, and parathyroid hormone were normal. However, laboratory examination's result shown that our patient has hypocalcemia, hyperphosphatemia, and parathyroid hormone deficiency and has a good response to the treatment. In addition, Fahr's disease generally has familial genetic predisposition, while the relatives of our patient have no similar symptoms. Finally, we have also considered genetic testing, but families refused to have a genetic test due to the financial difficulties. Therefore, the patient was more likely to have a diagnosis of idiopathic hypoparathyroidism.

This is, to our knowledge, the first report of idiopathic hypoparathyroidism in which initial symptoms were psychological and behavioral abnormalities. She was not correctly diagnosed until after thorough laboratory and neurological analysis in our department, in part as a result of the long, chronic course of the disease and the absence of symptoms typical of hypocalcemia. Our finding of intracranial calcification by routine computed tomography was critical in helping us arrive at the correct diagnosis rather than a misdiagnosis of primary mental disorders, which might have seemed reasonable given the rarity of idiopathic hypoparathyroidism relative to hypoparathyroidism secondary to surgery [2]. The present case may help clinicians avoid making such an error. In addition, clinicians should consider the possibility of idiopathic hypoparathyroidism if patients with mental disorders fail to respond to antipsychotic or antidepressant therapy, even if they did not show hypocalcemia before onset of psychiatric symptoms.

The literature contains some cases of secondary hypoparathyroidism in which the accompanying symptoms were mental disorders. Nearly all cases occurred as a result of surgery and hypomagnesemia induced by proton pump inhibitors (Table 1). Most of these patients 
Case Report Yanming Xu, PhD

\begin{tabular}{|l|l|l|l|l|l|}
\hline Table 1: Cases of secondary hypoparathyroidism involving psychological and behavioral abnormalities. \\
\hline Study & Age & Sex & Psychiatric manifestation & Initial manifestation & $\begin{array}{l}\text { Etiology of } \\
\text { hypoparathyroidism }\end{array}$ \\
\hline Lawlor [6], 1988 & & & Severe anxiety & Severe anxiety & $\begin{array}{l}\text { Ablation of a parathyroid } \\
\text { adenoma }\end{array}$ \\
\hline Anthony [4], $\mathbf{1 9 9 5}$ & $\mathbf{1 5}$ & Female & Abnormal behavioral changes & Abnormal behavioral changes & Hypomagnesemia \\
\hline Wodarz [8], $\mathbf{1 9 9 5}$ & $\mathbf{6 3}$ & Female & Musical hallucinations & Musical hallucinations & Post-thyroidectomy \\
\hline Thomas [5], 2007 & $\mathbf{6 8}$ & Female & Depression & $\begin{array}{l}\text { Paresthesias of the hand and } \\
\text { feet }\end{array}$ & Parathyroid surgery \\
\hline Regis [7], 2014 & $\mathbf{2 2}$ & Male & Depression & Periodic seizures & Chronic omeprazole use \\
\hline Present case & 53 & Female & $\begin{array}{l}\text { Psychological and behavioral } \\
\text { abnormalities }\end{array}$ & $\begin{array}{l}\text { Psychological and behavioral } \\
\text { abnormalities }\end{array}$ & Idiopathic \\
\hline
\end{tabular}

showed typical symptoms of hypocalcemia induced by hypoparathyroidism before onset of psychiatric symptoms. In this way, the present case significantly extends our understanding of the various clinical manifestations of hypoparathyroidism.

\section{Conclusion}

Patients with idiopathic hypoparathyroidism may initially present with psychological and behavioral abnormalities before other symptoms considered typical of the disease, such as hypocalcemia. Clinicians and psychiatrists should consider the possibility of idiopathic hypoparathyroidism during differential diagnosis of primary mental and behavioral disorders when a patient shows abnormal calcium and phosphate metabolism as well as intracranial calcification.

\section{Competing Interests}

The authors declare that they have no competing interests.

\section{Funding}

This work was funded by the Sichuan Key Project of Science and Technology (2010SZ0086).

\section{References}

1. Aggarwal S, Kailash S, Sagar R, et al. Neuropsychological dysfunction in idiopathic hypoparathyroidism and its relationship with intracranial calcification and serum total calcium. Eur. J. Endocrinol 168(6), 895-903 (2013).

2. Abate EG, Clarke BL. Review of Hypoparathyroidism. Front. Endocrinol (Lausanne) 7, 172 (2016).

3. Shoback DM, Bilezikian JP, Costa AG, et al. Presentation of Hypoparathyroidism: Etiologies and Clinical Features. J. Clin.
Endocrinol. Metab 101(6), 2300-2312 (2016).

4. Ang AW, Ko SM, Tan CH. Calcium, magnesium, and psychotic symptoms in a girl with idiopathic hypoparathyroidism. Psychosom. Med 57(3), 299-302 (1995).

5. Bohrer T, Krannich JH. Depression as a manifestation of latent chronic hypoparathyroidism. World. J. Biol. Psychiatry 8(1), 56-59 (2007).

6. Lawlor BA. Hypocalcemia, hypoparathyroidism, and organic anxiety syndrome. J. Clin. Psychiatry 49(8), 317-318 (1998).
7. Rosa RG, Barros AJ, de Lima AR, et al. Mood disorder as a manifestation of primary hypoparathyroidism: a case report. J. Med. Case. Rep 8, 326 (2014).

8. Wodarz N, Becker T, Deckert J. Musical hallucinations associated with postthyroidectomy hypoparathyroidism and symmetric basal ganglia calcifications. J. Neurol. Neurosurg. Psychiatry 58(6), 763-764 (1995).

9. Bilezikian JP, Brandi ML, Cusano NE, et al. Management of Hypoparathyroidism: Present and Future. J. Clin. Endocrinol. Metab 101(6), 2313-2324 (2016). 